\title{
LABYRINTHINE FISTULA IN CHOLESTEATOMA AND ITS MANAGEMENT-A RETROSPECTIVE STUDY
}

\begin{abstract}
Authors: Surendra Singh Baghel (1) Amit Keshri (2) Ravi Sankar Manogaran (3) Rajat Jain (4) Arulalan Mathailagan (5)

Authors Affiliations: (1) Senior Resident (2) Additional Professor (3) Associate Professor (5) PDCC, Neurootology, Department of Neurosurgery Sanjay Gandhi Post Graduate Institute of Medical Sciences, Lucknow (4) Assistant professor, Department of Otorhinolaryngology, King George's Medical University, Lucknow, Uttar Pradesh, India.
\end{abstract}

\begin{abstract}
Introduction- Labyrinthine fistula is one of the most common intra-temporal complications of cholesteatoma. Objective of study is (1) to evaluate the role of CT Scan in detecting labyrinthine fistulas, (2)to establish fact that fistula grade and location have no correlation with surgical approaches(CWU Vs CWD mastoidectomy approach),(3) to study the impact on hearing after treatment of labyrinthine fistula and(4) establish a management protocol to deal with labyrinthine fistula.
\end{abstract}

\section{MATERIAL AND METHODS}

Retrospective study done for 20 patients of labyrinthine fistula out of 149 cholesteatoma patients who underwent surgery for cholesteatoma in a tertiary care hospital between February 2015 to January 2020. Data collected for preoperative clinical signs and symptoms, HRCT temporal bone $0.5 \mathrm{~mm}$ cuts both axial and coronal cuts, Intra-operative fistula grade, site and surgical technique and pre and post operative audiometric outcomes.

\section{RESULTS}

Preoperative HRCT temporal predicted fistula in 15(75\%) patients. Using the Dornhoffer and Milewski classification, $15(75 \%)$ patients were identified as stage 1, 4(20\%) patients as stage II, and $1(5 \%)$ patient as stage III. Post operatively $4(20 \%)$ patients showed improvement,13(65\%) showed no change and $3(15 \%)$ showed deterioration in sensorineural hearing. There is statistically significant relationship between grade of labyrinthine fistula and the hearing outcome postoperatively ( $p$ value $=.036$ )

Conclusion- Clinical signs and symptoms of labyrinthine fistula are not diagnostic preoperatively. HRCT temporal bone is mandatory in making the diagnosis of labyrinthine fistula preoperatively. Choice of surgery between canal wall up(CWU) or canal wall down( CWD) mastoidectomy is independent of the type of fistula. Although there is an inverse correlation between fistula grade and post operative sensorineural hearing outcome yet overall preservation of hearing is good. Complete and meticulous removal of the cholesteatoma matrix over the fistula in a single staged procedure and its repair using temporalis fascia followed by bone dust is a safe and effective procedure to treat labyrinthine fistula.

\section{KEYWORDS}

Labyrinthine fistula, Lateral semicircular canal ,Cholesteatoma,High resolution computed tomography ,Bone conduction threshold

\section{DECLARATIONS}

Funding-Not applicable

Conflicts of intrest-none

Ethics approval-Taken by instutional ethics committee SGPGI lucknow ,IEC code:2020-160-IPEXP-21

Consent to participate-Not applicable

Consent for publication-Not applicable

Code availability-yes

Availability of data-yes 


\section{INTRODUCTION}

Labyrinthine fistula is a known complication of cholesteatoma. Erosion of labyrinthine block is caused by multiple factors like: pressure necrosis, ischemia, osteoclasts activation, reduced $\mathrm{pH}$ levels, inflammatory mediators related to infection and osteolytic enzymes [1]. About $87 \%$ of labyrinthine fistula are located over the lateral semicircular canal (LSCC) [2]. Preoperative clinical assessment is neither sensitive nor specific for diagnosing labrynthine fistula $[2,3]$. Prediction of labyrinthine fistula can be done by doing high resolution computed tomography (HRC) temporal bone preoperatively [4]. According to the depth of labyrinthine fistula Palva and Ramsay [5] gave 4 stage classification and Dornhoffer and Milewski [6] gave 3 stage classification. According to size sanna et al [7] divided fistula into 3 stages (small .5$1 \mathrm{~mm}$, medium 1-2 $\mathrm{mm}$ and large $>2 \mathrm{~mm}$ ) and Ikeda et al 8 divided labrynthine fistula into 2 stages small $(<3 \mathrm{~mm})$ and large group $(>3 \mathrm{~mm})$. Management of labyrinthine fistula is controversial, Broadly two techniques are advocated in literature : first complete removal of cholesteatoma matrix over fistula site with immediate repair, to eradicate potential source of bone resorption $[5,8,9]$ and second advocates leaving the matrix over fistula site undisturbed for maintenance of hearing and vestibular function $[10,11]$

Severe sensorineural hearing loss has been reported between $3 \%$ to $37 \%$ in patients who underwent surgical management of labyrinthine fistula [12-15]

The aim of the our study is to evaluate the role of HRCT temporal bone in detecting labyrinthine fistula preoperatively, to show that fistula grade and location have no correlation with surgical approaches (canal wall down(CWD) versus canal wall up (CWU)approaches), to study the impact on hearing after treatment of labyrinthine fistula and finally to establish a management protocol to deal with labyrinthine fistula.

\section{MATERIALS AND METHODS}

Retrospective study done in a tertiary care hospital between February 2015 to January 2020. We included 20 patients having single labrynthine fistula confirmed intra operatively out of 149 patients who underwent mastoidectomy (CWU or CWD) for cholesteatoma, irrespective of age and sex. Cholesteatoma with cochlear promontory fistula with profound hearing loss, multiple fistula, cholesteatoma with intracranial complication was excluded from study.

\section{Preoperative settings:}

Records of age, gender, diseased ear side, patient symptoms, audiometric analysis by PTA (pure tone audiometry) to evaluate hearing loss, endoscopic ear evaluation by $4 \mathrm{~mm} 0$ degree telescope, HRCT temporal bone axial and coronal $0.5 \mathrm{~mm}$ cuts to look for labyrinthine fistula were collected.

Audiometric data were based on the American Academy of Otolaryngology Head and Neck surgery guidelines at $0.5,1,2$, and $4 \mathrm{kHz}$. Pure tone averages were calculated on these 4 frequencies [16].

\section{Intraoperative settings:}

CWU or CWD mastoidectomy was done in all cases through post aural route. Labrynthine fistula identified and catergorized as per Dornhoffer and Milewski classification. Dornhoffer et al divided labrynthine fistula into 3 stages: stage I represents erosion of the bony labyrinth with intact endosteum; stage II is a true fistula with an opened perilymphatic space; and stage III is an opened perilymphatic space with concomitant involvement or destruction of the underlying membranous labyrinth. Stage II fistula is categorized further into stages Ila and Illb, but we did not subclassify stage II in this study.

Complete excision of cholesteatoma was done in all the cases along with repair of labrynthine fistula. Temporalis fascia followed by bone dust was used to repair labyrinthine fistula in all cases. All patients underwent excision of cholesteatoma and repair of labrynthine fistula in single stage. 


\section{Post operative settings:}

Post operative audiometric evaluation was done after 4 to 6 weeks of surgery. Averaged air and bone conduction (BC) thresholds were compared before surgery and after surgery. Postoperative averaged BC thresholds were classified in three groups: unchanged, improved hearing, decreased hearing.

We analyzed association between patients age, sex, location of labyrinthine fistula, disease duration, surgical approaches, bone conduction threshold change and the fistula stage using chisquare ,Fisher's exact test. ( $p$ value of $<0.05$ was considered significant)

\section{RESULTS}

20 (13.42\%)(11 males, 9 females) out of 149 patients of cholesteatoma presented with labyrinthine fistula. Age distribution ranged between 13-61 years (mean 31.65). As per Dornhoffer and Milewski classification, 15(75\%) patients were identified as stage 1, 4(20\%) patients as stage II, and $1(5 \%)$ patient as stage III. Primary surgery was performed in 17 cases out of 20. Three patients underwent revision surgery. (Table no 1)

Table No. 1: Summary of clinical and radiological features, operative findings and hearing results.

\begin{tabular}{|c|c|c|c|c|c|c|c|c|c|}
\hline \begin{tabular}{|c|} 
Case \\
no.
\end{tabular} & Sex & Age & $\begin{array}{c}\text { Semi } \\
\text { circular } \\
\text { canal }\end{array}$ & Stage & $\begin{array}{c}\text { Pre op } \\
\mathrm{BC}(\mathrm{dB}) \\
\text { threshold }\end{array}$ & $\begin{array}{l}\text { Post op } \\
\mathrm{BC}(\mathrm{dB}) \\
\text { threshold }\end{array}$ & $\begin{array}{l}\text { FN dehiscence } \\
\text { intraopeartively }\end{array}$ & $\begin{array}{c}\text { Pre } \\
\text { op } \\
\text { HRCT }\end{array}$ & Surgery \\
\hline 1 & $\mathrm{~F}$ & 36 & LSCC & 1 & 10 & 10 & - & - & CWDM $^{*}$ \\
\hline 2 & $F$ & 13 & LSCC & 1 & 10 & 8.75 & + & + & CWDM \\
\hline 3 & M & 19 & LSCC & 1 & 58.75 & 58.75 & - & - & CWDM $^{*}$ \\
\hline 4 & $F$ & 18 & LSCC & 2 & 55 & 90 & + & + & CWDM \\
\hline 5 & F & 49 & LSCC & 1 & 25 & 25 & + & + & CWUM \\
\hline 6 & $M$ & 59 & LSCC & 1 & 25 & 25 & + & + & CWDM \\
\hline 7 & $F$ & 22 & LSCC & 3 & 0 & 25 & + & + & CWUM \\
\hline 8 & $M$ & 21 & LSCC & 1 & 10 & 10 & + & + & CWUM \\
\hline 9 & $M$ & 17 & LSCC & 1 & 60 & 60 & + & + & CWDM $^{*}$ \\
\hline 10 & $M$ & 14 & LSCC & 1 & o & o & + & + & CWUM \\
\hline 11 & $F$ & 46 & LSCC & 1 & 15 & 15 & + & + & CWDM \\
\hline 12 & $M$ & 58 & LSCC & 2 & 25 & 15 & + & - & CWDM \\
\hline 13 & $M$ & 37 & LSCC & 1 & 40 & 40 & + & + & CWUM \\
\hline 14 & $F$ & 50 & LSCC & 2 & 10 & 10 & + & + & CWUM \\
\hline 15 & $\mathrm{~F}$ & 19 & LSCC & 1 & 40 & 40 & + & + & CWUM \\
\hline 16 & $M$ & 61 & LSCC & 1 & 60 & 60 & + & + & CWDM \\
\hline 17 & $M$ & 32 & LSCC & 1 & 10 & 55 & + & + & CWDM \\
\hline 18 & $M$ & 18 & LSCC & 2 & 20 & 15 & + & - & CWDM \\
\hline 19 & $\mathrm{~F}$ & 21 & LSCC & 1 & 27.5 & 2.5 & + & + & CWUM \\
\hline 20 & $M$ & 23 & PSCC & 1 & 15 & 15 & - & - & CWDM \\
\hline
\end{tabular}

LSCC-lateral semicircular canal, PSCC-posterior semicircular canal, BC-bone conduction CWUM-canal wall up mastoidectomy, CWDM canal wall down mastoidectomy

*-revision surgery

\section{Clinical assessment-}

\section{Preopeartively}

Otorrhoea and hearing loss was seen in all (100 $\%)$. Dizziness was seen in 13 patients (65\%), tinnitus in 3 patients (15\%), headache and vomiting in 3 patients (15\%).Duration of symptoms was between 6 to 40 yrs (mean 18.18 yrs). A positive fistula sign was present in only 2 patients (10\%). (Table no 1)

\section{Postoperatively:}

Follow up done for 6 months to 5 years(mean 2.1 years ). $40 \%$ patients complaints of dizziness in immediate post operative period, which was resolved within 2 weeks by labrynthine sedatives. No postoperative facial palsy or intracranial complications occurred.

\section{Audiological assessment-Pure tone audiometry(PTA) \\ Preoperative:}

Preoperative PTA showed conductive hearing loss in 10 patients $(50 \%)$ and mixed hearing loss in 10 patients (50\%).Pre operative averaged $A C$ threshold was $64.625 \mathrm{~dB}$ and $\mathrm{BC}$ threshold was $25.81 \mathrm{~dB}$.

\section{Postoperative:}

Postoperative averaged $A C$ threshold was 53.5 $\mathrm{dB}$ and $\mathrm{BC}$ threshold was $29 \mathrm{~dB}$.

Preoperative and postoperative $\mathrm{BC}$ thresholds were compared. 13(65\%) patients had stable hearing, 4(20\%) patients showed improvement and $3(15 \%)$ patients cases showed worsening. There was statistically significant relationship between labyrinthine fistula and hearing outcome (Pvalue $=0.036$ )

In stage I labyrinthine fistula (n-15)- 14 (93.33\%) patients showed no change or improvement and $1(6.67 \%)$ showed worsening.

In stage II labyrinthine fistula (n-4) - 3(75\%) \%) 
patients showed no change or improvement and $1(25 \%)$ showed worsening.

In stage III labyrinthine fistula(n-1) - 1(100\%) $\%)$ patients showed worsening of hearing.(Table no 2) so we observed that as the stage of fistula increased ,there are more chances of worsening of post operative cochlear functions.

Table No. 2 : Correlation of labyrinthine fistula grade with fistula sign, post operative $B C$ change, pre-operative HRCT prediction and surgical technique.

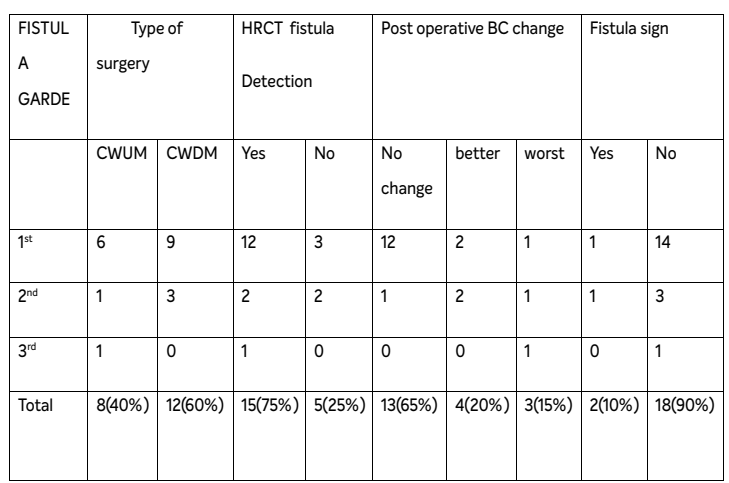

Hearing outcomes are not affected by surgical technique.

Table No 3 : Surgical technique versus post operative sensorineural hearing change.

\begin{tabular}{|c|c|c|c|c|}
\hline Surgery type & \multicolumn{3}{|c|}{ Post operative Bone conduction hearing } & \\
\hline & worse & better & No change & Total \\
\hline CWUM & 2 & 3 & 7 & 12 \\
\hline CWDM & 1 & 1 & 6 & 8 \\
\hline Total & $3(15 \%)$ & $4(20 \%)$ & $13(65 \%)$ & 20 \\
\hline
\end{tabular}

\section{Preoperative HRCT temporal bone prediction :}

Labyrinthine fistula was suspected in 15 cases(75\%).

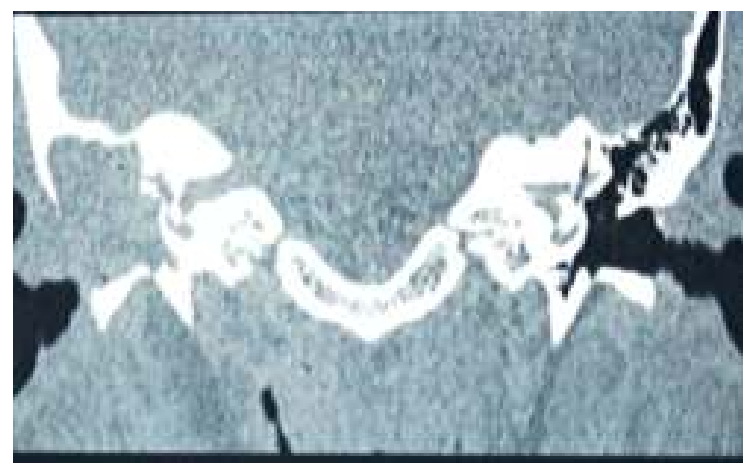

(a)

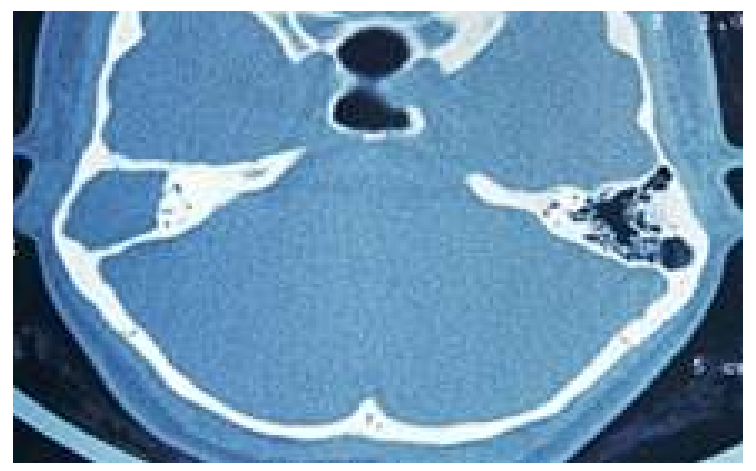

(b)

Fig 1- High resolution computed tomography scan temporal bone coronal (a) and axial (b) view showing right lateral semicircular canal fistula(white arrow).

There is no correlation between preoperative CT prediction and fistula grade.(Table no 2 )

\section{Intraoperative findings:}

Using the Dornhofferand Milewski classification, $75 \%$ were classified as stage 1, $20 \%$ as stage II,

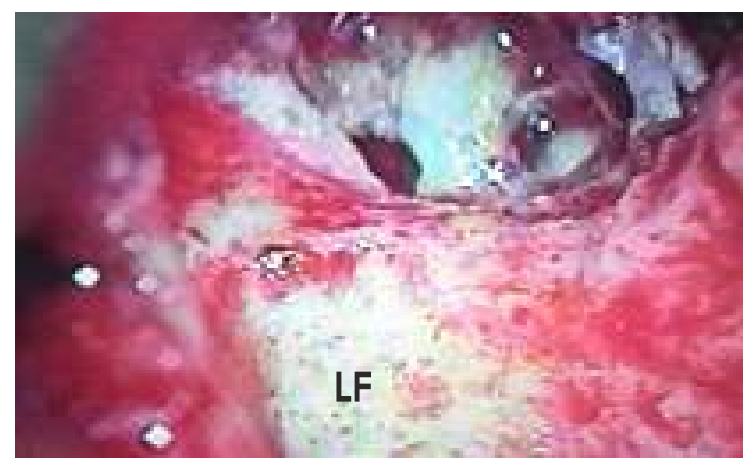


and $5 \%$ as stage III facial nerve was dehiscent in 17 cases(85\%). 19 out of 20 involving LSCC (95\%)and 1 involving PSCC(5\%).(Table no 1)

Fig 2- Intra operative picture right canal wall down mastoidectomy showing grade I labyrinthine fistula over lateral semicircular canal.

LF-lateral semicircular canal fistula.

\section{Surgical procedure (canal wall up or canal wall down)}

CWU mastoidectomy was performed in 8 (40\%) patients whereas CWD mastoidectomy was done in 12(60\%) patients, 3 of them were revision surgery (Table no 1). The choice of surgical technique was determined by status of posterior canal wall (eroded or intact), extension of disease, and size of mastoid and prior ear surgery. The choice of procedure was independent of the presence and grade of fistula. (Table no 2)

\section{DISCUSSION}

Labyrinthine fistula is one of the common complications of cholesteatoma. Incidence of labyrinthine fistula in literature varies between 3 to $16 \%$ with a average of $8 \%[17,18]$. In our study, the incidence of labrynthine fistula is $13.42 \%$. In 5 years, 20 out of 149 patients who were operated for cholesteatoma presented with labrynthine fistula. LSCC fistula was seen in 19 patients (95\%) and PSCC in 1 (5\%).LSCC is the most common location for labyrinthine fistula $[2,6,17,19]$ due to preferred anatomical location of cholesteatoma in concordance with our study.

The average age in our study was 33.65 years, ranging between 13 to 61 years, which is similar to that reported in the literature [18-20] .Preoperative symptoms like otorrhoea and hearing loss which occurred in $100 \%$ of the cases are non specific for labrynthine fistula and could be seen in chronic otitis media without labrynthine fistula.

Clinical signs and symptoms for labyrinthine fistula in cholesteatoma are positive fistula test and vertigo. Only $10 \%$ cases in our study found positive for fistula test, which was low as compared to a systemic review study by Copeland and Buchman et al [2] where they found positive fistula sign in 50\% patients. Discrepancy in results may be due to presence of cholesteatoma causing interruption of transmission of pressure from the external ear canal to the site of fistula. vertigo was present in $65 \%$ patients in our study compared to $45 \%$ as seen in study by Meyer et al 18 and $100 \%$ for Hakuba et al [21] . we observed that positivity of the fistula test was not in correlation with the fistula grade (Table no 2). Vertigo and positive fistula test are poor indicators of labyrinthine fistula, as seen in existing literature $[2,8,18,20]$. Tinnitus was present in $15 \%$ patients in our study similar to seen in study by Meyer et al [8] (16\%) . In our study there was no case with pre operative facial palsy with labrynthine fistula but some studies reported upto $10 \%$ of labrynthine fistula associated with pre operative facial nerve weakness $[1,8,22]$.

HRCT temporal bone was done in all case of cholesteatoma to see anatomy, extent of disease , rule out complication and to tailor our surgical approach. The sensitivity of the HRCT temporal to detect labyrinthine fistula is $75 \%$ ( Table no 1) in our study which is slightly lowered compared to current literature showing 85 to $100 \%$ sensitivity $[8,18,19]$. This could be due to poor quality of HRCT temporal bone film cuts done at peripheral diagnostic center and long duration between imaging and date of surgery. For better planning and results, HRCT temporal bone $0.5 \mathrm{~mm}$ axial and coronal cuts should be done within a period of one month from date of surgery.

In our study there is no correlation between fistula stage introperatively and pre operative HRCT scan detection sensitivity (Table no 2) .ikeda et al [8] found paradox results which showed preoperative HRCT detection sensitivity $70 \%$ in stage I fistula,94\% in stage II fistula and $100 \%$ in stage III fistula. Stephenson et al[19]showed $100 \%$ sensitivity and specificity in fistula detection irrespective of fistula stage. 
Stephenson et al [19] also demostrated identification of membranous fistula versus bony fistula with $66 \%$ sensitivity and $71 \%$ specificity respectively on preoperative HRCT temporal bone.

The management of labyrinthine fistula is controversial. Whether to do complete or partial removal of cholesteatoma matrix over fistula site/ do it in single stage or two stage/ approach of surgery (CWU Mastoidectomy vs CWD Mastoidectomy)?

Ritter and Freeman left matrix over fistula to avoid opening of labyrinth and preserve hearing $[10,11]$. Plava et al[23] reported a case of dead ear after 3 years of matrix preserving surgery due to suppurative labyrinthitis. Some authors left cholesteatoma matrix over fistula site during primary surgery and removed it during the second stage after 6 months $[7,24,25]$ to obtain better functional results with reduced risks of hearing loss. Sanna et al [7] presented concept of "reverse metaplasia" i.e mucosalization of cholesteatoma matrix in $69 \%$ of post operative cases during reexploration. This could be due to restoration of normal ventilation of middle ear following disease removal in primary surgery. In a systemic review analysis Lim et al [26] found that rate of hearing preservation were similar in both staged and unstaged procedure.

Recent literature favor total eradication of cholesteatoma in a one-stage surgery $[8,18,19]$ because complete cholesteatoma matrix removal results in reduction of bone resorption and reduced risk of suppurative labyrinthitis, $[2,21]$ but it has potential to cause postoperative sensorineural hearing loss, but according to Copeland and Buchman et al [2] and Lim et al [26] both techniques (total removal or preservation of matrix over fistula) are alike in preserving hearing.

In our study, we performed single stage surgery in all cases with complete removal of cholesteatoma matrix and immediate repair using temporalis fascia followed by bone dust at the end of procedure irrespective of fistula grading.

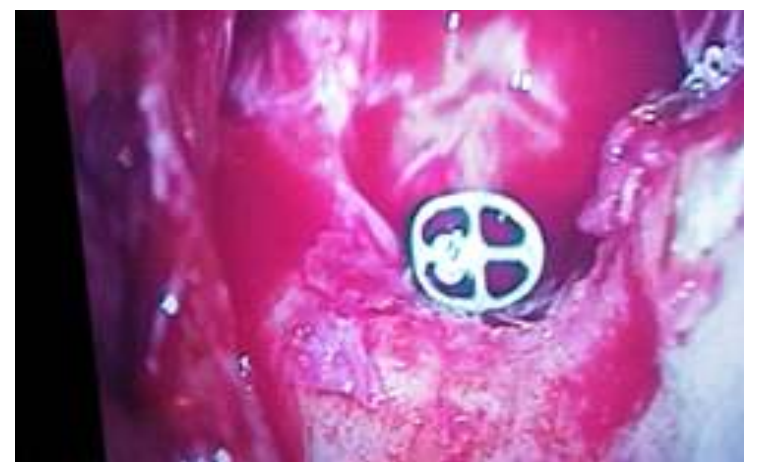

RLF

Fig 3- Right ear canal wall down mastoidectomy with titanium TORP ossiculoplasty and lateral semicircular canal fistula repaired with bone dust and temporalis fascia. RLF-repaired lateral semicircular canal fistula, TORP-total ossicular replacement prosthesis

Freeman, Hakuba and Abramson et al $[11,21,27]$ strongly believed that if labyrinthine fistula is present, radical or modified radical mastoidectomy is the preferred option. Sheey et al [17] advocated classical modified radical mastoidectomy if labyrinthine fistula was suspected in only hearing ear. Sanna et al [12] advocated canal wall down surgery in case of only hearing ear with multiple fistula or revision surgery. Sanna et al [12] showed that hearing outcome are not affected by surgical technique which is concomitant to our study. (Table no 3 ).

Most studies shows that choice of the technique(CWUM vs CWDM) adopted was dependent on the extent of disease, mastoid size, the history of prior ear surgery but not on the presence of labyrinthine fistula and its grade $[12,18,19]$ which is again in concomitant to our study.

We advocate possible preferred technique to be canal wall up mastoidectomy, to maintain natural anatomy, avoid cavity related problem and minimize the need for long term care.

In our study post operatively hearing improvement was seen in $20 \%$ patients, hearing was preserved in $65 \%$ cases and in 15\% cases there was deterioration in sensorineural hearing 
which was similar to existing literature [8, 18, 20]. Intraoperative labrynthine fistula staging correlated with the outcome of $\mathrm{SNHL}$ (sensorineural hearing loss) postoperatively. In stage I labrynthine fistula (n-15) 14(93.33\%) patients showed no change or improvement in hearing status postoperatively and 1(6.67\%) patient showed detoriation in hearing status postopeartively. In stage II labrynthine fistula (n4) $3(75 \%)$ patients showed no change or improvement in hearing status postoperatively and $1(25 \%)$ patient showed detoriation of hearing status postoperatively. In stage III labrynthine fistula (n-1) 100\% patients showed detoriation of hearing status postoperatively. So we would like to conclude that as the stage of fistula increases ,there are more chances of detoriation of post operative cochlear functions. By classifying labrynthine fistula stage intraopeartively we can asses post operative hearing status. Some series do not show any correlation between labrynthine fistula grade and post operative sensorineural hearing outcome $[8,18,20]$.

\section{CONCLUSION}

Labyrinthine fistula is a common complication of extended cholesteatoma with most common site being LSCC. Since preoperatively sign and symptoms of labyrinthine fistula are not diagnostic, HRCT temporal bone is of utmost importance in making its diagnosis. The choice of canal wall up mastoidectomy or canal wall down mastoidectomy is determined by the characteristics of cholesteatoma, but is independent of the presence of labrynthine fistula and its grade. Complete and meticulous removal of cholesteatoma matrix over the labrynthine fistula, followed by its repair in a single-staged procedure is a safe and effective procedure. Intraopeartive staging of fistula can be used to prognosticate post operative hearing outcome of patient. We have tried to give a management protocol for management of labrynthine fistula with cholesteatoma for good outcomes.

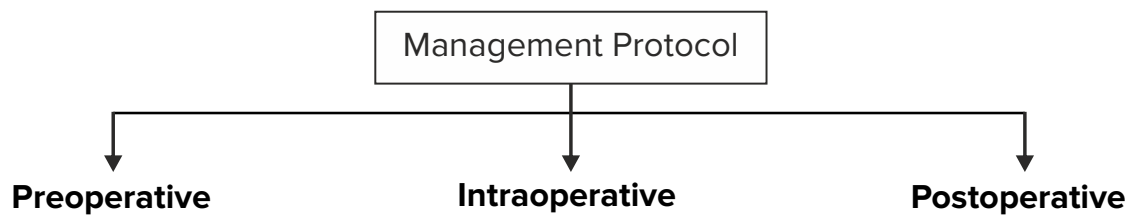

- High suspicion of labyrinthine fistula in every case of cholesteatoma, irrespective of clinical signs and symptoms.

- Imaging - $0.5 \mathrm{~mm}$ thick slice HRCT temporal bone both axial and coronal cut.
- Post operative systemic antibiotics coverage for at least 1 week.

- PTA after 4-6 weeks of surgery.

- Vestibular sedative in early post operative period.

\begin{tabular}{|c|c|}
\hline $\begin{array}{l}\text { Surgical technique selection criteria } \\
\text { - Extent of cholesteatoma } \\
\text { - Mastoid size and pneumatization } \\
\text { - Status of posterior canal wall } \\
\text { - Complete and safe removal of matrix }\end{array}$ & $\begin{array}{l}\text { Matrix management } \\
\text { 1. Complete and non traumatic removal in single stage by- } \\
\text { - Meticulous dissection under higher magnification by senior } \\
\text { - } \quad \text { Nurgeon at the end of surgery. } \\
\text { - Continous saline irrigation. } \\
\text { 2. Immediate repair with already prepared temporalis fascia } \\
\text { followed by bone dust irrespective of grade of fistula. }\end{array}$ \\
\hline
\end{tabular}




\section{REFERENCES}

1. Soda-Merhy A, Betancourt-Sua'rez MA (2000) Surgical treatment of labyrinthine fistula caused by cholesteatoma. Otolaryngol Head Neck Surg 122(5):739-742

2. Am J Otolaryngol. 2003 Jan-Feb;24(1):51-60. Management of labyrinthine fistulae in chronic ear surgery. Copeland BJ(1), Buchman CA.

3. Portier F, Lescanne E, Racy E, Nowak C, Lamblin B, Bobin S (2005) Studies of labyrinthine cholesteatoma-related Wstulas: report of 22 cases. J Otolaryngol 34(1):1-6

4. Fuse T, Tada Y, Aoyagi M, Sugai Y (1996) CT detection of facial canal dehiscence and semicircular canal Wstula: comparison with surgical Wndings. J Comput Assist Tomogr 20(2):221-224

5. Palva T, Ramsay H (1989) Treatment of labyrinthine fistula. Arch Otolaryngol Head Neck Surg 115:804-806

6. Dornhoffer JL, Milewski C. Management of the open labyrinth. Otolaryngol Head Neck Surg. 1995 Mar;112(3):410-4. PubMed PMID: 7870441

7. Sanna M, Zini C, Bacciu S, Scandellari R, Delogu P, Jemmi G (1984) Management of the labyrinthine Wstula in cholesteatoma surgery. ORL J Otorhinolaryngol Relat Spec 46(3):165-172

8. Ikeda R, Kobayashi T, Kawase T et al (2012) Risk factors for deterioration of bone conduction hearing in cases of labyrinthine fistula caused by middle ear cholesteatoma. Ann OtolRhinol Laryngol 121:162-16

9. Gersdorff MCH, Debaty ME, Tomasi JP (2006) Pathophysiology of cholesteatoma. Rev Laryngol-Otol-Rhinol 127:115-119

10. Ritter FN (1970) Chronic suppurative otitis media and the pathologic labyrinthine fistula. Laryngoscope 80:1025-1035

11. Freeman P (1978) Fistula of the lateral semicircular canal. ClinOtolaryngolAllied Sci 3(3):315-321

12. Sanna M, Zini C, Gamoletti R et al (1988) Closed versus open technique in the management of labyrinthine fistulae. Am J Otolc 9:470-475

13. Ostri B, Bak-Pedersen K. Surgical management of labyrinthine fistulae in chronic otitis media with cholesteatoma by a one-stage closed technique. ORL J
Otorhinolaryngol Relat Spec 1989;51:295-9.

14. Tos M. Treatment of labyrinthine fistulas by a closed technique.ORL J Otorhinolaryngol Relat Spec 1975;37:41-7.

15. McCabe BF. Labyrinthine fistula in chronic mastoiditis. Ann Otol Rhinol Laryngol 1983;93(suppl 112):138-41.

16. Committee on Hearing and Equilibrium guidelines for the evaluation of results of treatment of conductive hearing loss (1995) American Academy of Otolaryngology-Head and Neck SurgeryFoundation, Inc. Otolaryngol Head Neck Surg 113:186-187

17. Sheehy JL, Brackmann DE (1979) Cholesteatoma surgery: management of the labyrinthine fistula-a report of 97 cases. Laryngoscope 89:78-87

18. Meyer, A., Bouchetemblé, P., Costentin, B. et al. Lateral semicircular canal fistula in cholesteatoma: diagnosis and management. Eur Arch Otorhinolaryngol 273, 2055-2063

19. Stephenson M-F, Saliba I (2011) Prognostic indicators of hearing after complete resection of cholesteatoma causing a labyrinthine fistula. Eur Arch Oto-RhinoLaryngol 268:1705-1711

20. Ueda Y, Kurita T, Matsuda Y, et al. (2009) Surgical treatment of labyrinthine fistula in patients with cholesteatoma. J LaryngolOtolSuppl 64-67

21. Hakuba N, Hato N, Shinomori Y, Sato H, Gyo K. Labyrinthine fistula as a late complication of middle ear surgery using the canal wall down technique. OtolNeurotol 2002;23:832

22. Romanet $P$, Duvillard $C$, Delouane $M$ et al (2001) Labyrinthine fistulae and chol esteatom a. A n n OtolaryngolChirCervicofac118(3):181-186

23. Palva T, Karja J, Palva A (1971) Opening of the labyrinth during chronic ear surgery. Arch Otolaryngol 93(1):75-78

24. Glasscock, M.E.: Results in cholesteatoma surgery. Cholesteatoma, first international conference, pp. 401-403 (Aesculapius Publishing Company, Birmingham 1977

25. Law KP, Smyth GD, Kerr AG (1975) Fistulae of the labyrinth treated by staged combined approach tympanoplasty. J Laryngol Otol 89(5):471-478

26. Lim, John \& Gangal, Anupriya \& Gluth, Michael. ( 2017 ). Surgery for Cholesteatomatous Labyrinthine Fistula: A 
Systematic Review. Annals of Otology, Rhinology \& Laryngology. 000348941668319 . 10.1177/0003489 416683193

27. Abramson M, Harker LA, McCabe BF (1974) Labyrinithine fistula complicating chronic suppurative otitis media. Arch Otolaryngol 100(2):141-142

\section{${ }^{*}$ Corresponding Author:}

Dr. Amit Keshri, MS (ENT)

Additional Professor (Neuro-otology)

Department of Neurosurgery,

Sanjay Gandhi Post Graduate Institute of Medical Sciences

Lucknow, Uttar Pradesh, India, 226014

Phone Number: 07275721669,08004904461

Email ID:amitkeshri2000@yahoo.com 